\title{
Error bound analysis for vector equilibrium problems with partial order provided by a polyhedral cone
}

\author{
Nguyen Van Hung ${ }^{1}$ (D) Vicente $\mathrm{Novo}^{2}$ (D) Vo Minh $\operatorname{Tam}^{3}$
}

Received: 1 September 2020 / Accepted: 1 July 2021 / Published online: 24 July 2021

(c) The Author(s) 2021, corrected publication October 2021

\begin{abstract}
The aim of this paper is to establish new results on the error bounds for a class of vector equilibrium problems with partial order provided by a polyhedral cone generated by some matrix. We first propose some regularized gap functions of this problem using the concept of $\mathcal{G}_{A}$-convexity of a vector-valued function. Then, we derive error bounds for vector equilibrium problems with partial order given by a polyhedral cone in terms of regularized gap functions under some suitable conditions. Finally, a real-world application to a vector network equilibrium problem is given to illustrate the derived theoretical results.
\end{abstract}

Keywords Vector equilibrium problem - Vector network equilibrium problem $\cdot$ Regularized gap function · Error bound · Polyhedral cone

Mathematics Subject Classification 90C30 - 90C26 · 49J52

\section{Introduction}

It is well known that the theory of equilibrium problems is a generalization of various problems related to optimization such as variational inequalities, complementarity problems and optimization problems, etc. Equilibrium problems have remarkable applications in the fields of mechanics, network analysis, transportation, finance, economics, operations research and

Vicente Novo

vnovo@ind.uned.es

Nguyen Van Hung

nvhungmath@gmail.com

Vo Minh Tam

vmtam@dthu.edu.vn

1 Department of Scientific Fundamentals, Posts and Telecommunications Institute of Technology, Ho Chi Minh City, Vietnam

2 Departamento de Matemática Aplicada, E.T.S.I. Industriales, Universidad Nacional de Educación a Distancia, calle Juan del Rosal 12, Ciudad Universitaria, 28040 Madrid, Spain

3 Department of Mathematics, Dong Thap University, Cao Lanh City, Vietnam 
optimization. For details, we refer the readers to works $[9,13,18,20,25,26]$ and the references therein.

A scalar equilibrium problem is defined as follows:

(EP): find $x^{*} \in K$ such that $F\left(x^{*}, y\right) \geq 0$, for all $y \in K$,

where $K$ is a given set, $F: K \times K \rightarrow \mathbb{R}$ is a bifunction satisfying $F(x, x)=0$ for all $x \in K$. The problem (EP) is also known under the name of equilibrium problem in the works of Blum and Oettli [4], Muu and Oettli [30], or Ky Fan equilibrium problem in [11].

The error bound of a certain problem is known as an upper estimate of the distance between an arbitrary feasible point and the solution set. It has played a crucial role from the point of view of theoretical analysis as well as to study the convergence of iterative algorithms for solving optimization problems, complementarity problems, variational inequalities and equilibrium problems. In 2003, based on the study of gap functions of (EP), Mastroeni [29] established global error bounds for (EP) under the assumption of strong monotonicity of $F$. The theory of gap functions in [29] is a generalization of the gap functions for variational inequalities considered in the literature. The notion of gap functions was first introduced by Auslender (1976) [2] to reformulate the variational inequality into an equivalent optimization problem:

$$
\Upsilon(x)=\sup _{y \in K}\langle h(x), x-y\rangle
$$

subject to $x \in K \subset \mathbb{R}^{n}$, where $h: \mathbb{R}^{n} \rightarrow \mathbb{R}^{n}$, and $\langle\cdot, \cdot\rangle$ is the scalar product in $\mathbb{R}^{n}$. However, in general, the Auslender gap functions are not differentiable. To overcome this non-differentiability, Fukushima [12], Yamashita and Fukushima [39] proposed the notion of regularized gap functions for variational inequalities:

$$
\Upsilon_{\alpha}(x)=\sup _{y \in K}\left\{\langle h(x), x-y\rangle-\alpha\|x-y\|^{2}\right\},
$$

where $\alpha$ is a nonnegative parameter. Moreover, Yamashita and Fukushima [39] also established the regularized function of Moreau-Yosida type as follows:

$$
\Gamma \Upsilon_{\alpha}, \lambda(x)=\inf _{z \in K}\left\{\Upsilon_{\alpha}(z)+\lambda\|x-z\|^{2}\right\},
$$

where $\lambda$ is a positive parameter. Using gap functions in forms of the Fukushima regularization and the Moreau-Yosida regularization, Yamashita and Fukushima [39] established global error bounds for general variational inequalities. Thereafter, many regularized gap functions and error bounds have been studied for various kinds of equilibrium problems and variational inequalities, see e.g., [1,3,19,21-24,27,35] and the references therein for a more detailed discussion of interesting topics. In particular, Khan and Chen [27] established regularized gap functions and error bounds for generalized mixed vector equilibrium problems under the partial order introduced by the usual positive cone in finite dimensional spaces. Anh et al. [1] and Hung et al. [23] developed the results in [27] for generalized mixed vector equilibrium problems of strong types and mixed vector variational inequalities, respectively, whose final space is partially ordered by a infinite dimensional cone.

On the other hand, the study of linear inequalities has been applied to mathematical programming and econometrics (see Stoer and Witzgall [34]). This study has led to investigate a special class of cones, the polyhedral cones (cf. Definition 2.1). The theory of polyhedral cones is considered extensively. For more details, we refer the interested readers to [5,14,34]. Using properties of polyhedral convex cones associated to the matrices, Chegancas and Burgat [6] established sufficient conditions for asymptotic stability of a linear discrete-time-varing 
system. A class of linear complementarity problems over a polyhedral cone was considered by Zhang et al. [40]. They also designed a Newton-type algorithm for solving it. Very recently, Gutiérrez et al. $[15,16]$ characterized the several kind of exact and approximate efficient solutions of a class of multiobjective optimization problems with partial order provided by a polyhedral cone. From a computational point of view, they showed that the results in $[15,16]$ based on the ordering cone generated by some matrix are attractive. Using the partial order considered in [16], Hai et al. [17] continued to investigating vector equilibrium problems with a polyhedral ordering cone. In [17], variants of the Ekeland variational principle for a type of approximate proper solutions of those vector equilibrium problems were also provided. However, to the best of our knowledge, up to now, there is no paper devoted to error bounds for vector equilibrium problems and vector network equilibrium problems, whose final space is finite dimensional partially ordered by a polyhedral cone generated by some matrix.

Inspired by the works above, in this paper, we establish some new results on the error bounds for a class of vector equilibrium problems with a partial order defined by a polyhedral cone generated by some matrix based on regularized gap functions in forms of the Fukushima regularization and the Moreau-Yosida regularization introduced in [39] by using the concept of $\mathcal{G}_{A}$-convexity. Finally, we illustrate the main results by an application to a vector network equilibrium problem for which we establish analysing error bounds.

The paper is structured as follows. In Sect. 2 we introduce the framework, notations and definitions which are needed along the paper. Based on a partial order provided by a polyhedral cone, in Sect. 3 we propose some regularized gap functions for a class of vector equilibrium problems under some suitable conditions. Then, the error bound analysis for these problems in terms of regularized gap functions is studied. An application to a vector network equilibrium problem is given to illustrate our main theoretical results in Sect. 4. Finally, some conclusions are stated in Sect. 5.

\section{Preliminaries and notations}

We first introduce a class of vector equilibrium problems with partial order provided by a polyhedral cone generated by some matrix $A$ as follows:

$\operatorname{VEP}\left(\mathcal{C}, F, \mathcal{G}_{A}\right)$ : Find $x^{*} \in \mathcal{C}$ such that

$$
F\left(x^{*}, y\right) \notin-\operatorname{int}\left(\mathcal{G}_{A}\right),
$$

for all $y \in \mathcal{C}$, where $\mathcal{C}$ is a nonempty closed and convex subset of a real normed space $X$, $A=\left(a_{i j}\right)$ is a real matrix with $p$ rows and $m$ columns with the positive integers $p, m$ such that $p \geq m$ and $\operatorname{rank}(A)=m, \mathcal{G}_{A}$ is a polyhedral cone generated by $A$ (cf. Definition 2.1) such that $\mathcal{G}_{A}$ has non-empty interior, $F: \mathcal{C} \times \mathcal{C} \rightarrow \mathbb{R}^{m}$ is a vector-valued function such that $F(x, x)=0$ for all $x \in \mathcal{C}$. In case of $\mathcal{C}=X$ being $X$ is a nontrivial complete metric space, the problem $\operatorname{VEP}\left(\mathcal{C}, F, \mathcal{G}_{A}\right)$ was mentioned in [17].

Next, we recall some basic concepts and their properties. Throughout the paper, let $\mathbb{R}^{p}$ be the $p$-dimensional Euclidean space and

$$
\mathbb{R}_{+}^{p}=\left\{\left(a_{1}, \ldots, a_{p}\right) \in \mathbb{R}^{p}: a_{i} \geq 0, \quad \forall i=1, \ldots, p\right\} .
$$

For any two vectors $a=\left(a_{1}, \ldots, a_{p}\right)^{\top}$ and $b=\left(b_{1}, \ldots, b_{p}\right)^{\top}, a, b \in \mathbb{R}^{p}$, we define the relationships between vectors as follows:

$$
\begin{aligned}
& a \leq b \text { if and only if } a_{i} \leq b_{i} \text { for all } i \in\{1, \ldots, p\}, \\
& a<b \text { if and only if } a_{i}<b_{i} \text { for all } i \in\{1, \ldots, p\}
\end{aligned}
$$


A nonempty set $\mathcal{G} \subset \mathbb{R}^{p}$ is a cone if $\lambda x \in \mathcal{G}$ for all $x \in \mathcal{G}$ and $\lambda \geq 0$. A cone $\mathcal{G}$ is said to be pointed if $\mathcal{G} \cap-\mathcal{G}=\{\mathbf{0}\}$, where $\mathbf{0}=(0, \ldots, 0)^{\top} \in \mathbb{R}^{p}$.

As usual, a hyperplane in $\mathbb{R}^{p}$ is a set associated with some $(a, b) \in \mathbb{R}^{p} \times \mathbb{R}, a \neq \mathbf{0}$, and defined as $\left\{x \in \mathbb{R}^{p}:\langle a, x\rangle=b\right\}$. The closed half-space of $\mathbb{R}^{p}$ is a set associated with some $(a, b) \in \mathbb{R}^{p} \times \mathbb{R}, a \neq \mathbf{0}$, and defined as $\left\{x \in \mathbb{R}^{p}:\langle a, x\rangle \leq b\right\}$. A set $P \subset \mathbb{R}^{p}$ is said to be a polyhedral set if it can be expressed as the intersection of a finite family of closed half-spaces or hyperplanes.

Proposition 2.1 (see [32]) The following statements are equivalent for a set $\mathcal{G} \subset \mathbb{R}^{m}$ :

(i) $\mathcal{G}$ is a polyhedral cone;

(ii) $\mathcal{G}$ has a representation of the form

$$
\mathcal{G}=\left\{x \in \mathbb{R}^{m}:\left\langle a_{i}, x\right\rangle \leq 0, \forall i=1, \ldots, p\right\},
$$

for some positive integer $p$ and some $a_{i} \in \mathbb{R}^{m}, i=1, \ldots, p$.

Denote the set of all real matrices with $p$ rows and $m$ columns by $\mathbb{R}^{p \times m}$.

Definition 2.1 (see [10]) Let $A \in \mathbb{R}^{p \times m}$. Then

$$
\mathcal{G}_{A}=\left\{x \in \mathbb{R}^{m}: A x \geq \mathbf{0}\right\},
$$

which is called a cone generated by $A$.

The cone $\mathcal{G}_{A}$ is polyhedral, and so it is also convex and closed.

Proposition 2.2 (see [31], Proposition 4 and Proposition 5) Let $A \in \mathbb{R}^{p \times m}$. Then

(i) The cone $\mathcal{G}_{A}$ defined by (4) is pointed if and only if $\operatorname{rank}(A)=m(p \geq m)$.

(ii) If the matrix A has no zero rows, then

$$
\operatorname{int}\left(\mathcal{G}_{A}\right)=\left\{x \in \mathbb{R}^{m}: A x>\mathbf{0}\right\}
$$

Lemma 2.1 (see [38], Lemma 1) Let $A \in \mathbb{R}^{p \times m}$. If $\mathcal{G}_{A}=\{\mathbf{0}\}$, then $\operatorname{rank}(A)=m$ and $p>m$.

Let $A \in \mathbb{R}^{p \times m}$ be a given matrix. The mapping defined by the matrix $A$ is also denoted by $A$, where $A: \mathbb{R}^{m} \rightarrow \mathbb{R}^{p}$ defined by $x \mapsto A x$ (or $A(x)$ ) is a bounded linear mapping.

Proposition 2.3 (see [33], Proposition 4.1) Let $A$ be a mapping defined by a matrix $A \in$ $\mathbb{R}^{p \times m}$. Assume that the set $\left\{x \in \mathbb{R}^{m}: A x \geq \mathbf{0}\right\}$ is a pointed cone, or, equivalently, that $\operatorname{rank}(A)=m$ and $p \geq m$. Then, the following statements hold:

(i) the mapping $A$ is injective,

(ii) the image of the set $\left\{x \in \mathbb{R}^{m}: A x \geq \mathbf{0}\right\}$ under the mapping $A$ is a convex cone included in $\mathbb{R}_{+}^{p}$,

(iii) if $p=m$, then the image of the space $\mathbb{R}^{m}$ under the mapping $A$ is $\mathbb{R}^{p}$ and the image of the cone $\left\{x \in \mathbb{R}^{m}: A x \geq \mathbf{0}\right\}$ is $\mathbb{R}_{+}^{p}$,

(iv) if $p>m$, then the image of the space $\mathbb{R}^{m}$ under the mapping $A$ is a proper subset of $\mathbb{R}^{p}$ and the image of the cone $\left\{x \in \mathbb{R}^{m}: A x \geq \mathbf{0}\right\}$ is a proper subset of $\mathbb{R}_{+}^{p}$.

To end this section, we derive a useful remark to convert the problem $\operatorname{VEP}\left(\mathcal{C}, F, \mathcal{G}_{A}\right)$ into a usual vector equilibrium problem, which is easier to handle from a computational point of view. 
Remark 2.1 Suppose that the function $F: \mathcal{C} \times \mathcal{C} \rightarrow \mathbb{R}^{m}$ is defined by

$$
F(x, y)=\left(F_{1}(x, y), \ldots, F_{m}(x, y)\right)^{\top} \in \mathbb{R}^{m}
$$

for all $x, y \in \mathcal{C}$. Let $A \circ F$ denote the composition of the mapping defined by the matrix $A$ with the function $F$. Hence, $A \circ F: \mathcal{C} \times \mathcal{C} \rightarrow \mathbb{R}^{p}$ is defined by

$$
A \circ F=\left((A \circ F)_{1}, \ldots,(A \circ F)_{p}\right)^{\top} \text { and }(A \circ F)(x, y)=A(F(x, y)), \quad \forall x, y \in \mathcal{C} .
$$

Then, thanks to Proposition 2.2(ii), Proposition 2.3(ii) and the linearity of mapping $A$, we can show that (1) is equivalent to

$$
(A \circ F)\left(x^{*}, y\right) \notin-\operatorname{int}\left(\mathbb{R}_{+}^{p}\right) .
$$

\section{Main results}

In this section, we shall introduce the notion of the $\mathcal{G}_{A}$-convexity of a vector-valued function based on the partial order provided by a polyhedral cone. Then regularized gap functions and error bounds for the problem $\operatorname{VEP}\left(\mathcal{C}, F, \mathcal{G}_{A}\right)$ will be investigated by using the property of the $\mathcal{G}_{A}$-convexity and some suitable assumptions.

Throughout the paper, unless other specified, let $X$ be a real normed space with norm $\|\cdot\|$ and $\mathcal{C}$ be a nonempty closed and convex subset of $X$. For a fixed subset $D \subset X$ and $a \in X$, the distance between the point $a$ and the set $D$ is $d(a, D)=\inf _{d \in D}\{\|a-d\|\}$. Denote the solution set of the problem $\operatorname{VEP}\left(\mathcal{C}, F, \mathcal{G}_{A}\right)$ by $\mathbf{S}\left(\mathcal{C}, F, \mathcal{G}_{A}\right)$. We always assume that $\mathbf{S}\left(\mathcal{C}, F, \mathcal{G}_{A}\right)$ is a nonempty set.

Definition 3.1 A real function $\rho: \mathcal{C} \rightarrow \mathbb{R}$ is said to be a gap function for the problem $\operatorname{VEP}\left(\mathcal{C}, F, \mathcal{G}_{A}\right)$ if the following properties hold:

(a) $\rho(x) \geq 0$ for all $x \in \mathcal{C}$;

(b) for any $x^{*} \in \mathcal{C}, \rho\left(x^{*}\right)=0$ if and only if $x^{*}$ is a solution of the problem $\operatorname{VEP}\left(\mathcal{C}, F, \mathcal{G}_{A}\right)$.

For each fixed constant $\gamma>0$, we now consider the following function $\Omega_{\gamma}^{\varrho}: \mathcal{C} \rightarrow \mathbb{R}$ defined by

$$
\begin{aligned}
\Omega_{\gamma}^{\varrho}(x) & =\sup _{y \in \mathcal{C}}\left(-\max _{1 \leq i \leq p}\left\{(A \circ F)_{i}(x, y)\right\}-\gamma \varrho(x, y)\right) \\
& =\sup _{y \in \mathcal{C}}\left(-\max _{1 \leq i \leq p}\left\{\sum_{k=1}^{m} a_{i k} F_{k}(x, y)\right\}-\gamma \varrho(x, y)\right),
\end{aligned}
$$

where $\varrho: \mathcal{C} \times \mathcal{C} \rightarrow \mathbb{R}_{+}$is a continuously differentiable function, which satisfies the following property with the associated constants $\beta \geq 2 \alpha>0$.

$$
\left(\mathcal{H}_{\varrho}\right): \quad \text { For all } x, y \in \mathcal{C}, \alpha\|x-y\|^{2} \leq \varrho(x, y) \leq(\beta-\alpha)\|x-y\|^{2} .
$$

Definition 3.2 For each $k \in\{1, \ldots, m\}$, let $\varphi_{k}: \mathcal{C} \rightarrow \mathbb{R}$ be a function. A function $\varphi:=$ $\left(\varphi_{1}, \ldots, \varphi_{m}\right)$ defined by $\varphi(x)=\left(\varphi_{1}(x), \ldots, \varphi_{m}(x)\right)$ is said to be $\mathcal{G}_{A}$-convex if, for all $x, y \in \mathcal{C}$ and $\lambda \in[0,1]$,

$$
\lambda \varphi(x)+(1-\lambda) \varphi(y)-\varphi(\lambda x+(1-\lambda) y) \in \mathcal{G}_{A} .
$$


Remark 3.1 (i) The condition (7) is equivalent to

$$
A(\lambda \varphi(x)+(1-\lambda) \varphi(y)-\varphi(\lambda x+(1-\lambda) y)) \in \mathbb{R}_{+}^{p}
$$

that is, for any $i \in\{1, \ldots, p\}$,

$$
\sum_{k=1}^{m} a_{i k}\left[\lambda \varphi_{k}(x)+(1-\lambda) \varphi_{k}(y)-\varphi_{k}(\lambda x+(1-\lambda) y)\right] \geq 0 .
$$

(ii) Moreover, if $a_{i k} \geq 0$ and $\varphi_{k}$ is a convex function for all $i \in\{1, \ldots, p\}$ and $k \in$ $\{1, \ldots, m\}$, then it is easy to see that the above inequality holds and so $\varphi$ is $\mathcal{G}_{A}$-convex. However, the reverse implication does not hold, that is, assume that $\varphi$ is $\mathcal{G}_{A}$-convex. Then it is not necessary that the function $\varphi_{k}$ is convex for all $k \in\{1, \ldots, m\}$. The following example shows the converse is not true.

Example 3.1 Let $X=\mathbb{R}, \mathcal{C}=[0,2], p=m=2$ and the matrix $A \in \mathbb{R}^{p \times m}$ be defined by $A=\left(\begin{array}{ll}1 & 2 \\ 1 & 0\end{array}\right)$. Then, $\operatorname{rank}(A)=2$ and we have

$$
\begin{aligned}
\mathcal{G}_{A} & =\left\{x=\left(x_{1}, x_{2}\right)^{\top} \in \mathbb{R}^{2}: A x \geq \mathbf{0}\right\} \\
& =\left\{x=\left(x_{1}, x_{2}\right)^{\top} \in \mathbb{R}^{2}: x_{1}+2 x_{2} \geq 0 \text { and } x_{1} \geq 0\right\} .
\end{aligned}
$$

Let $\varphi=\left(\varphi_{1}, \varphi_{2}\right)^{\top}: \mathcal{C} \rightarrow \mathbb{R}^{2}$ be defined as follows:

$$
\varphi(x)=\left(\varphi_{1}(x), \varphi_{2}(x)\right)^{\top}=\left(3 x^{2}+1,-x^{2}\right)^{\top} .
$$

For each $i \in\{1,2\}$, it is easy to check that the inequality (8) holds for all $x, y \in \mathcal{C}$ and $\lambda \in[0,1]$. Thus, it follows from Remark 3.1(i) that $\varphi$ is $\mathcal{G}_{A}$-convex on $\mathcal{C}$. However, the function $\varphi_{2}$ defined by $\varphi_{2}(x)=-x^{2}$ is not convex on $\mathcal{C}$.

Proposition 3.1 Let $F$ be $\mathcal{G}_{A}$-convex in the second component and $\varrho$ satisfy the condition $\left(\mathcal{H}_{\varrho}\right)$. Then for any $\gamma>0$, the function $\Omega_{\gamma}^{\varrho}$ defined by (6) is finite-valued on $\mathcal{C}$.

Proof For each $\gamma>0$, it follows from (6) that

$$
\begin{aligned}
\Omega_{\gamma}^{\varrho}(x) & =\sup _{y \in \mathcal{C}}\left(-\max _{1 \leq i \leq p}\left\{\sum_{k=1}^{m} a_{i k} F_{k}(x, y)\right\}-\gamma \varrho(x, y)\right) \\
& =-\inf _{y \in \mathcal{C}}\left(\max _{1 \leq i \leq p}\left\{\sum_{k=1}^{m} a_{i k} F_{k}(x, y)\right\}+\gamma \varrho(x, y)\right) \\
& \leq-\inf _{y \in \mathcal{C}}\left(\max _{1 \leq i \leq p}\left\{\sum_{k=1}^{m} a_{i k} F_{k}(x, y)\right\}+\gamma \alpha\|x-y\|^{2}\right)
\end{aligned}
$$

( $\varrho$ satisfies the condition $\left(\mathcal{H}_{\varrho}\right)$ ).

Since $F$ is $\mathcal{G}_{A}$-convex in the second component, for all $i \in\{1, \ldots, p\}, y_{1}, y_{2} \in \mathcal{C}$ and $\lambda \in[0,1]$, by Remark 3.1(i), we have

$$
\sum_{k=1}^{m} a_{i k}\left[\lambda F_{k}\left(x, y_{1}\right)+(1-\lambda) F_{k}\left(x, y_{2}\right)-F_{k}\left(x, \lambda y_{1}+(1-\lambda) y_{2}\right)\right] \geq 0,
$$


that is,

$$
\sum_{k=1}^{m} a_{i k} F_{k}\left(x, \lambda y_{1}+(1-\lambda) y_{2}\right) \leq \lambda \sum_{k=1}^{m} a_{i k} F_{k}\left(x, y_{1}\right)+(1-\lambda) \sum_{k=1}^{m} a_{i k} F_{k}\left(x, y_{2}\right) .
$$

This implies that for all $i \in\{1, \ldots, p\}$, the function $y \mapsto \sum_{k=1}^{m} a_{i k} F_{k}(x, y)$ is convex and so the function

$$
y \mapsto \max _{1 \leq i \leq p}\left\{\sum_{k=1}^{m} a_{i k} F_{k}(x, y)\right\}+\gamma \alpha\|x-y\|^{2}
$$

is strongly convex. Since $\mathcal{C}$ is a closed and convex set, the function (9) has a unique minimizer over $\mathcal{C}$. Thus $\Omega_{\gamma}^{\varrho}$ is finite-valued on $\mathcal{C}$.

Theorem 3.1 Suppose that $F$ is $\mathcal{G}_{A}$-convex in the second component and $\varrho$ satisfies the condition $\left(\mathcal{H}_{\varrho}\right)$. Then the function $\Omega_{\gamma}^{\varrho}$ defined by (6) for any $\gamma>0$ is a gap function for $\operatorname{VEP}\left(\mathcal{C}, F, \mathcal{G}_{A}\right)$.

Proof (a) For each $\gamma>0$ fixed, since $F_{k}(x, x)=0$ and $\varrho(x, x)=0$ for all $k \in\{1, \ldots, m\}$ and $u \in \mathcal{C}$, we have

$$
\Omega_{\gamma}^{\varrho}(x) \geq-\max _{1 \leq i \leq p}\left\{\sum_{k=1}^{m} a_{i k} F_{k}(x, x)\right\}-\gamma \varrho(x, x)=0
$$

(b) Suppose $x^{*} \in \mathcal{C}$ such that $\Omega_{\gamma}^{\varrho}\left(x^{*}\right)=0$, namely,

$$
\sup _{y \in \mathcal{C}}\left(-\max _{1 \leq i \leq p}\left\{\sum_{k=1}^{m} a_{i k} F_{k}\left(x^{*}, y\right)\right\}-\gamma \varrho\left(x^{*}, y\right)\right)=0 .
$$

This implies

$$
-\max _{1 \leq i \leq p}\left\{\sum_{k=1}^{m} a_{i k} F_{k}\left(x^{*}, y\right)\right\} \leq \gamma \varrho\left(x^{*}, y\right), \forall y \in \mathcal{C} .
$$

Equivalently,

$$
\min _{1 \leq i \leq p}\left\{-\sum_{k=1}^{m} a_{i k} F_{k}\left(x^{*}, y\right)\right\} \leq \gamma \varrho\left(x^{*}, y\right), \forall y \in \mathcal{C} .
$$

Then there exits $i_{0} \in\{1, \ldots, p\}$ such that

$$
-\sum_{k=1}^{m} a_{i_{0} k} F_{k}\left(x^{*}, y\right) \leq \gamma \varrho\left(x^{*}, y\right)
$$

for all $y \in \mathcal{C}$. For any $x \in \mathcal{C}$ and $\lambda \in(0,1)$, we set $y_{\lambda}:=\lambda x^{*}+(1-\lambda) x \in \mathcal{C}$, and so

$$
-\sum_{k=1}^{m} a_{i_{0} k} F_{k}\left(x^{*}, y_{\lambda}\right) \leq \gamma \varrho\left(x^{*}, y_{\lambda}\right) \text {. }
$$

It follows from the condition $\left(\mathcal{H}_{\varrho}\right)$ that

$$
\varrho\left(x^{*}, y_{\lambda}\right) \leq(\alpha-\beta)\left\|x^{*}-y_{\lambda}\right\|^{2}=(1-\lambda)^{2}(\alpha-\beta)\left\|x^{*}-x\right\|^{2} .
$$


By the $\mathcal{G}_{A}$-convexity of $y \mapsto F\left(x^{*}, y\right)$ and $F_{k}\left(x^{*}, x^{*}\right)=0$ for all $k \in\{1, \ldots, m\}$, we have

$$
\sum_{k=1}^{m} a_{i_{0} k}\left[\lambda F_{k}\left(x^{*}, x^{*}\right)+(1-\lambda) F_{k}\left(x^{*}, x\right)-F_{k}\left(x^{*}, y_{\lambda}\right)\right] \geq 0 .
$$

The above inequality becomes

$$
-\sum_{k=1}^{m} a_{i_{0} k} F_{k}\left(x^{*}, y_{\lambda}\right) \geq-(1-\lambda) \sum_{k=1}^{m} a_{i_{0} k} F_{k}\left(x^{*}, x\right) .
$$

Having in mind relations (10)-(12), it gives

$$
-(1-\lambda) \sum_{k=1}^{m} a_{i_{0} k} F_{k}\left(x^{*}, x\right) \leq(1-\lambda)^{2}(\alpha-\beta)\left\|x^{*}-x\right\|^{2} .
$$

Hence, we obtain

$$
-\sum_{k=1}^{m} a_{i_{0} k} F_{k}\left(x^{*}, x\right) \leq(1-\lambda)(\alpha-\beta)\left\|x^{*}-x\right\|^{2}
$$

for all $x \in \mathcal{C}$. In (13), letting $\lambda \rightarrow 1^{-}$, it implies

$$
\begin{aligned}
\sum_{k=1}^{m} a_{i_{0} k} F_{k}\left(x^{*}, x\right) \geq 0, \forall x \in \mathcal{C} & \Longleftrightarrow(A \circ F)\left(x^{*}, x\right) \notin-\operatorname{int}\left(\mathbb{R}_{+}^{p}\right), \forall x \in \mathcal{C} \\
& \Longleftrightarrow F\left(x^{*}, x\right) \notin-\operatorname{int}\left(\mathcal{G}_{A}\right), \forall x \in \mathcal{C},
\end{aligned}
$$

that is, $x^{*} \in \mathbf{S}\left(\mathcal{C}, F, \mathcal{G}_{A}\right)$.

Conversely, suppose that $x^{*}$ is a solution of $\operatorname{VEP}\left(\mathcal{C}, F, \mathcal{G}_{A}\right)$, that is, $x^{*} \in \mathcal{C}$ and

$$
F\left(x^{*}, y\right) \notin-\operatorname{int}\left(\mathcal{G}_{A}\right), \forall y \in \mathcal{C} \Longleftrightarrow(A \circ F)\left(x^{*}, y\right) \notin-\operatorname{int}\left(\mathbb{R}_{+}^{p}\right), \forall y \in \mathcal{C} .
$$

Hence, for every $y \in \mathcal{C}$, there exists $i_{0}(y) \in\{1, \ldots, p\}$ such that

$$
(A \circ F)_{i_{0}}\left(x^{*}, y\right) \geq 0 \text {, i.e., } \sum_{k=1}^{m} a_{i_{0} k} F_{k}\left(x^{*}, y\right) \geq 0 .
$$

Therefore,

$$
\sup _{y \in \mathcal{C}}\left(-\max _{1 \leq i \leq p}\left\{\sum_{k=1}^{m} a_{i k} F_{k}\left(x^{*}, y\right)\right\}-\gamma \varrho\left(x^{*}, y\right)\right) \leq 0 .
$$

It follows from the above inequality that $\Omega_{\gamma}^{\varrho}\left(x^{*}\right) \leq 0$. Since $\Omega_{\gamma}^{\varrho}\left(x^{*}\right) \geq 0$, one has $\Omega_{\gamma}^{\varrho}\left(x^{*}\right)=0$. The proof is completed.

Remark 3.2 Recently, several regularized gap functions for various kinds of vector equilibrium problems with partial order provided by the cone $\mathbb{R}_{+}^{m}$ have been studied. For instance, we reconsider a special case of the vector equilibrium problem in Khan and Chen [27], which consists in finding $x^{*} \in \mathcal{C}$ such that

$$
F\left(x^{*}, y\right)=\left(F_{1}\left(x^{*}, y\right), \ldots, F_{m}\left(x^{*}, y\right)\right)^{\top} \notin-\operatorname{int}\left(\mathbb{R}_{+}^{m}\right), \quad \forall y \in \mathcal{C} .
$$


Then, to establish the regularized gap function for problem (14), all component functions $y \mapsto F_{1}(x, y), y \mapsto F_{2}(x, y), \ldots, y \mapsto F_{m}(x, y)$ are imposed to be convex on $\mathcal{C}$ (see [27, Theorem 3.2]).

Meanwhile, our Theorem 3.1 used the characteristic of the $\mathcal{G}_{A}$-convexity in terms of a perturbation of the matrix $A$, that is, the convexity assumption of all component functions $F_{1}, F_{2}, \ldots, F_{m}$ in the gap function is not required.

Lemma 3.1 Assume that all assumptions of Theorem 3.1 hold and, further, that $\mathcal{C}$ is a compact set, $F_{k}$ is continuous for all $k \in\{1, \ldots, m\}$ and $\varrho$ satisfies the condition $\left(\mathcal{H}_{\varrho}\right)$. Then, for each $\gamma>0$, the gap function $\Omega_{\gamma}^{\varrho}$ is continuous on $\mathcal{C}$.

Proof Since $F_{k}$ is continuous for each $k \in\{1, \ldots, m\}$, and $\varrho$ is continuous, we have that the function $h: \mathcal{C} \times \mathcal{C} \rightarrow \mathbb{R}$ defined by

$$
\left.h(x, y)=-\max _{1 \leq i \leq p}\left\{\sum_{k=1}^{m} a_{i k} F_{k}(x, y)\right\}-\gamma \varrho(x, y)\right\}
$$

is continuous in $\mathcal{C} \times \mathcal{C}$. Moreover, $\mathcal{C}$ is a compact set, so the function $\Omega_{\gamma}^{\varrho}$ defined by

$$
\Omega_{\gamma}^{\varrho}(x)=\sup _{y \in \mathcal{C}} h(x, y)
$$

is continuous on $\mathcal{C}$. This completes the proof.

Now, we propose a gap function based on the Moreau-Yosida regularization of $\Omega_{\gamma}^{\varrho}$ as follows:

$$
\Delta_{\Omega_{\gamma}^{\varrho}, \delta}^{\psi}(x)=\inf _{u \in \mathcal{C}}\left\{\Omega_{\gamma}^{\varrho}(u)+\delta \psi(x, u)\right\},
$$

where $\psi: \mathcal{C} \times \mathcal{C} \rightarrow \mathbb{R}_{+}$is a continuously differentiable function, which satisfies the following property with the associated constants $\theta \geq 2 \eta>0$.

$$
\left(\mathcal{H}_{\psi}\right): \quad \text { For all } x, y \in \mathcal{C}, \eta\|x-y\|^{2} \leq \psi(x, y) \leq(\theta-\eta)\|x-y\|^{2} .
$$

$\Delta_{\Omega_{\gamma}^{\varrho}, \delta}^{\psi}$ defined by (15) is rewritten as follows:

$$
\Delta_{\Omega_{\gamma}, \delta}^{\psi}=\inf _{u \in \mathcal{C}}\left[\sup _{y \in \mathcal{C}}\left(-\max _{1 \leq i \leq p}\left\{\sum_{k=1}^{m} a_{i k} F_{k}(u, y)\right\}-\gamma \varrho(u, y)\right)+\delta \psi(x, u)\right] .
$$

Theorem 3.2 Suppose that all conditions of Lemma 3.1 hold, and assume further that $\psi$ satisfies the condition $\left(\mathcal{H}_{\psi}\right)$. Then the function $\Delta_{\Omega_{\gamma}{ }^{\varrho}, \delta}^{\psi}$ defined by (15) for any $\gamma, \delta>0$ is a gap function for $\operatorname{VEP}\left(\mathcal{C}, F, \mathcal{G}_{A}\right)$.

Proof (a) For any $\theta, \tau>0$, it is easy to show that $\Delta_{\Omega_{\gamma}^{\varrho}, \delta}^{\psi}(x) \geq 0$ for all $x \in \mathcal{C}$.

(b) Assume that $x^{*} \in \mathbf{S}\left(\mathcal{C}, F, \mathcal{G}_{A}\right)$. It follows from Theorem 3.1 that $\Omega_{\gamma}^{\varrho}\left(x^{*}\right)=0$ and so

$$
\begin{aligned}
\Delta_{\Omega_{\gamma}^{\varrho}, \delta}^{\psi}\left(x^{*}\right) & =\inf _{u \in \mathcal{C}}\left\{\Omega_{\gamma}^{\varrho}(u)+\delta \psi\left(x^{*}, u\right)\right\} \\
& \leq \Omega_{\gamma}^{\varrho}\left(x^{*}\right)+\delta \psi\left(x^{*}, x^{*}\right)=0 .
\end{aligned}
$$

Since $\Delta_{\Omega_{\gamma}^{\ell}, \delta}^{\psi}\left(x^{*}\right) \geq 0$, we get $\Delta_{\Omega_{\gamma}^{\varrho}, \delta}^{\psi}\left(x^{*}\right)=0$. 
Conversely, if $\Delta_{\Omega_{\gamma}, \delta}^{\psi}\left(x^{*}\right)=0$, i.e,

$$
\inf _{u \in \mathcal{C}}\left\{\Omega_{\gamma}^{\varrho}(u)+\delta \psi\left(x^{*}, u\right)\right\}=0 .
$$

Then, for each $n$, there is a $u_{n} \in \mathcal{C}$ such that

$$
\Omega_{\gamma}^{\varrho}\left(u_{n}\right)+\delta \psi\left(x^{*}, u_{n}\right)<\frac{1}{n} .
$$

Since $\psi$ satisfies the condition $\left(\mathcal{H}_{\psi}\right)$, it follows from (17) that

$$
0 \leq \Omega_{\gamma}^{\varrho}\left(u_{n}\right)+\delta \eta\left\|x^{*}-u_{n}\right\|^{2}<\frac{1}{n} .
$$

This implies that $\Omega_{\gamma}^{\varrho}\left(u_{n}\right) \rightarrow 0$ and $\left\|x^{*}-u_{n}\right\| \rightarrow 0$, as $n \rightarrow+\infty$, i.e., $\Omega_{\gamma}^{\varrho}\left(u_{n}\right) \rightarrow 0$ and $u_{n} \rightarrow x^{*}$, as $n \rightarrow+\infty$. Thanks to Lemma 3.1, the continuity of $\Omega_{\gamma}^{\varrho}$ holds, and so

$$
\Omega_{\gamma}^{\varrho}\left(x^{*}\right)=\lim _{n \rightarrow+\infty} \Omega_{\gamma}^{\varrho}\left(u_{n}\right)=0 .
$$

Therefore, we get $x^{*} \in \mathbf{S}\left(\mathcal{C}, F, \mathcal{G}_{A}\right)$. This completes the proof.

Now, we give the following example to illustrate all the assumptions of Theorems 3.1 and 3.2 are satisfied.

Example 3.2 Let $X=\mathbb{R}, \mathcal{C}=\left[0, e^{2}\right], p=m=2$ and the matrix $A \in \mathbb{R}^{p \times m}$ be defined by

$$
A=\left(\begin{array}{ll}
1 & 0 \\
\frac{1}{2} & \frac{1}{2}
\end{array}\right) \text {. }
$$

Then, $\operatorname{rank}(A)=2$ and we have

$$
\begin{aligned}
\mathcal{G}_{A} & =\left\{x=\left(x_{1}, x_{2}\right)^{\top} \in \mathbb{R}^{2}: A x \geq \mathbf{0}\right\} \\
& =\left\{x=\left(x_{1}, x_{2}\right)^{\top} \in \mathbb{R}^{2}: x_{1} \geq 0 \text { and } x_{1}+x_{2} \geq 0\right\} .
\end{aligned}
$$

Let $\varrho, \psi: \mathcal{C} \times \mathcal{C} \rightarrow \mathbb{R}_{+}$and $F=\left(F_{1}, F_{2}\right)^{\top}: \mathcal{C} \times \mathcal{C} \rightarrow \mathbb{R}^{2}$ be defined as follows:

$$
\begin{aligned}
\varrho(x, y) & =\psi(x, y)=\frac{1}{2}\|x-y\|^{2}=\frac{1}{2}(x-y)^{2}, \\
F(x, y) & =\left(F_{1}(x, y), F_{2}(x, y)\right)^{\top}, \\
F_{1}(x, y) & =y^{2}+2 x y-3 x^{2}, \\
F_{2}(x, y) & =y^{2}+3 x y-4 x^{2} .
\end{aligned}
$$

This implies that

$$
\begin{aligned}
(A \circ F)(x, y) & =A(F(x, y)) \\
& =\left(F_{1}(x, y), \frac{1}{2} F_{1}(x, y)+\frac{1}{2} F_{2}(x, y)\right)^{\top} \\
& =\left(y^{2}+2 x y-3 x^{2}, y^{2}+\frac{5}{2} x y-\frac{7}{2} x^{2}\right)^{\top} .
\end{aligned}
$$

By Remark 2.1, the problem $\operatorname{VEP}\left(\mathcal{C}, F, \mathcal{G}_{A}\right)$ is equivalent to finding $x \in \mathcal{C}$ such that

$$
(A \circ F)(x, y) \notin-\operatorname{int}\left(\mathbb{R}_{+}^{2}\right), \forall y \in \mathcal{C},
$$


that is, finding $x \in\left[0, e^{2}\right]$ such that

$$
\left(y^{2}+2 x y-3 x^{2}, y^{2}+\frac{5}{2} x y-\frac{7}{2} x^{2}\right)^{\top} \notin-\operatorname{int}\left(\mathbb{R}_{+}^{2}\right), \forall y \in\left[0, e^{2}\right] .
$$

Hence, it is easy to see that $x=0$ is the solution of the problem $\operatorname{VEP}\left(\mathcal{C}, F, \mathcal{G}_{A}\right)$. Thus, we have $\mathbf{S}\left(\mathcal{C}, F, \mathcal{G}_{A}\right)=\{0\}$.

By the convexity of $\mathbb{R} \ni y \mapsto F_{1}(x, y)$ and $\mathbb{R} \ni y \mapsto F_{2}(x, y)$ for all $x \in \mathbb{R}$, we can show that $\mathbb{R} \ni y \mapsto F(x, y)$ is $\mathcal{G}_{A}$-convex [by Remark 3.1(ii)]. Moreover, $\varrho$ is a continuously differentiable function and satisfies condition $\left(\mathcal{H}_{\varrho}\right)$ with $\beta=1, \alpha=\frac{1}{2}$. Therefore, all the assumptions of Theorem 3.1 hold. Then for any $\gamma>0$, the function $\Omega_{\gamma}^{\varrho}$ defined by (6) is a gap function for $\operatorname{VEP}\left(\mathcal{C}, F, \mathcal{G}_{A}\right)$. For example, we take $\gamma=1$, and we obtain

$$
\begin{aligned}
\Omega_{\gamma}^{\varrho}(x) & =\sup _{y \in \mathcal{C}}\left(-\max _{1 \leq i \leq p}\left\{(A \circ F)_{i}(x, y)\right\}-\gamma \varrho(x, y)\right) \\
& =\max _{y \in\left[0, e^{2}\right]}\left(-\max \left\{y^{2}+2 x y-3 x^{2}, y^{2}+\frac{5}{2} x y-\frac{7}{2} x^{2}\right\}-\frac{1}{2}(x-y)^{2}\right) \\
& =\max _{y \in\left[0, e^{2}\right]}\left(\frac{3}{2}\left(2 x^{2}-x y-y^{2}\right)\right) \\
& =3 x^{2} .
\end{aligned}
$$

Hence, $\Omega_{\gamma}^{\varrho}$ is a gap function for $\operatorname{VEP}\left(\mathcal{C}, F, \mathcal{G}_{A}\right)$.

By a similar argument, all the assumptions of Theorem 3.2 are satisfied with $\theta=1$ and $\eta=\frac{1}{2}$. Then for any $\gamma, \delta>0$, the function $\Delta_{\Omega_{\gamma}, \delta}^{\psi}$ defined by (15) is a gap function for $\operatorname{VEP}\left(\mathcal{C}, F, \mathcal{G}_{A}\right)$. For example, we take $\gamma=\theta=1$, and we have

$$
\begin{aligned}
\Delta_{\Omega_{\gamma}, \delta}^{\psi}(x) & =\inf _{u \in \mathcal{C}}\left\{\Omega_{\gamma}^{\varrho}(u)+\delta \psi(x, u)\right\} \\
& =\min _{u \in\left[0, e^{2}\right]}\left\{3 u^{2}+\frac{1}{2}(x-u)^{2}\right\}=\frac{3}{7} x^{2} .
\end{aligned}
$$

Remark 3.3 As mentioned before there is no result concerning regularized gap functions for the problem $\operatorname{VEP}\left(\mathcal{C}, F, \mathcal{G}_{A}\right)$. Thus, our results Theorems 3.1 and 3.2 are new in establishing regularized gap functions for vector equilibrium problems, whose final space is finite dimensional and partially ordered by a polyhedral cone generated by some matrix. However, if $m=p=1, A=(1), X=\mathbb{R}^{n}, F(x, y)=F_{1}(x, y)=\langle H(x), y-x\rangle$, $\varrho(x, y)=\psi(x, y)=\|x-y\|^{2}$ for all $x, y \in \mathcal{C}, H: \mathcal{C} \rightarrow \mathbb{R}^{n}$, then the functions $\Omega_{\gamma}^{\varrho}$ and $\Delta_{\Omega_{\gamma}, \delta}^{\psi}$ reduce to gap functions in forms of the Fukushima regularization and the MoreauYosida regularization introduced in [39], respectively for variational inequalities. Hence, in this sense, Theorems 3.1 and 3.2 extend the corresponding known results in [39].

To establish error bounds for the problem $\operatorname{VEP}\left(\mathcal{C}, F, \mathcal{G}_{A}\right)$, we now impose the following hypotheses:

$\left(\mathcal{H}_{F}\right)_{1}: \bigcap_{i=1}^{p} \mathcal{K}_{i}:=\left\{x \in \mathcal{C}: \sum_{k=1}^{m} a_{i k} F_{k}(x, y) \geq 0, \forall y \in \mathcal{C}\right\} \neq \emptyset ;$

$\left(\mathcal{H}_{F}\right)_{2}$ : There exists $\xi>0$ such that, for each $(x, y) \in \mathcal{C} \times \mathcal{C}$,

$$
F(x, y)+F(y, x)+\xi\|x-y\|^{2} e \in-\mathcal{G}_{A},
$$

where $e=(1,1, \ldots, 1)^{\top} \in \mathbb{R}^{m}$; 
$\left(\mathcal{H}_{A}\right)$ : The matrix $A \in \mathbb{R}^{p \times m}$ with $A=\left(a_{i j}\right)$ satisfies that

$$
\min _{1 \leq i \leq p}\left\{\sum_{k=1}^{m} a_{i k}\right\}>0 .
$$

Next, we establish error bounds for vector equilibrium problems with partial order provided by a polyhedral cone based on the regularized gap functions studied above.

Theorem 3.3 Suppose that all assumptions of Theorem 3.1 are satisfied and the conditions $\left(\mathcal{H}_{F}\right)_{1},\left(\mathcal{H}_{F}\right)_{2}$ and $\left(\mathcal{H}_{A}\right)$ hold. Then, for each $x \in \mathcal{C}$ and $\gamma>0$ satisfying

$$
\min _{1 \leq i \leq p}\left\{\sum_{k=1}^{m} a_{i k}\right\} \xi-\gamma(\beta-\alpha)>0,
$$

we have

$$
d\left(x, \mathbf{S}\left(\mathcal{C}, F, \mathcal{G}_{A}\right)\right) \leq \sqrt{\frac{\Omega_{\gamma}^{\varrho}(x)}{\min _{1 \leq i \leq p}\left\{\sum_{k=1}^{m} a_{i k}\right\} \xi-\gamma(\beta-\alpha)}} .
$$

Proof Let $x^{*} \in \mathbf{S}\left(\mathcal{C}, F, \mathcal{G}_{A}\right)$. For each $x \in \mathcal{C}$, we have

$$
\begin{aligned}
\Omega_{\gamma}^{\varrho}(x) & =\sup _{y \in \mathcal{C}}\left(-\max _{1 \leq i \leq p}\left\{\sum_{k=1}^{m} a_{i k} F_{k}(x, y)\right\}-\gamma \varrho(x, y)\right) \\
& \geq-\max _{1 \leq i \leq p}\left\{\sum_{k=1}^{m} a_{i k} F_{k}\left(x, x^{*}\right)\right\}-\gamma \varrho\left(x, x^{*}\right) .
\end{aligned}
$$

Without loss of generality, we assume that there exists $i^{*} \in\{1, \ldots, p\}$ such that

$$
\max _{1 \leq i \leq p}\left\{\sum_{k=1}^{m} a_{i k} F_{k}\left(x, x^{*}\right)\right\}=\sum_{k=1}^{m} a_{i * k} F_{k}\left(x, x^{*}\right)
$$

and so, (19) gives that

$$
\Omega_{\gamma}^{\varrho}(x) \geq-\sum_{k=1}^{m} a_{i^{*} k} F_{k}\left(x, x^{*}\right)-\gamma \varrho\left(x, x^{*}\right) .
$$

It follows from the condition $\left(\mathcal{H}_{F}\right)_{2}$ that

$$
A\left(F\left(x, x^{*}\right)+F\left(x^{*}, x\right)+\xi\left\|x-x^{*}\right\|^{2} e\right) \in-\mathbb{R}_{+}^{p},
$$

which implies

$$
\sum_{k=1}^{m} a_{i^{*} k}\left[F_{k}\left(x, x^{*}\right)+F_{k}\left(x^{*}, x\right)+\xi\left\|x-x^{*}\right\|^{2}\right] \leq 0,
$$

and so

$$
\begin{aligned}
-\sum_{k=1}^{m} a_{i * k} F_{k}\left(x, x^{*}\right)-\sum_{k=1}^{m} a_{i^{*} k} F_{k}\left(x^{*}, x\right) & \geq \sum_{k=1}^{m} a_{i * k} \xi\left\|x-x^{*}\right\|^{2} \\
& \geq \min _{1 \leq i \leq p}\left\{\sum_{k=1}^{m} a_{i k}\right\} \xi\left\|x-x^{*}\right\|^{2} .
\end{aligned}
$$



i.e.,

Since the condition $\left(\mathcal{H}_{F}\right)_{1}$ holds, without loss of generality, we assume that $x^{*} \in \mathcal{K}_{i^{*}}$,

$$
\sum_{k=1}^{m} a_{i * k} F_{k}\left(x^{*}, x\right) \geq 0 .
$$

Moreover, it follows from the property $\left(\mathcal{H}_{\varrho}\right)$ that

$$
-\varrho\left(x, x^{*}\right) \geq-(\beta-\alpha)\left\|x-x^{*}\right\|^{2} .
$$

Employing (20)-(23), we obtain

$$
\Omega_{\gamma}^{\varrho}(x) \geq\left(\min _{1 \leq i \leq p}\left\{\sum_{k=1}^{m} a_{i k}\right\} \xi-\gamma(\beta-\alpha)\right)\left\|x-x^{*}\right\|^{2} .
$$

This implies that

$$
\left\|x-x^{*}\right\| \leq \sqrt{\frac{\Omega_{\gamma}^{\varrho}(x)}{\min _{1 \leq i \leq p}\left\{\sum_{k=1}^{m} a_{i k}\right\} \xi-\gamma(\beta-\alpha)}},
$$

that is,

$$
d\left(x, \mathbf{S}\left(\mathcal{C}, F, \mathcal{G}_{A}\right)\right) \leq\left\|x-x^{*}\right\| \leq \sqrt{\frac{\Omega_{\gamma}^{\varrho}(x)}{\min _{1 \leq i \leq p}\left\{\sum_{k=1}^{m} a_{i k}\right\} \xi-\gamma(\beta-\alpha)}}
$$

and the proof is completed.

Theorem 3.4 Suppose that the assumptions of Theorems 3.2 and 3.3 hold. Then, for each $x \in \mathcal{C}$, we have

$$
d\left(x, S\left(\mathcal{C}, F, \mathcal{G}_{A}\right)\right) \leq \sqrt{\frac{2 \Delta_{\Omega_{\gamma}, \delta}^{\psi}(x)}{\min \left\{\min _{1 \leq i \leq p}\left\{\sum_{k=1}^{m} a_{i k}\right\} \xi-\gamma(\beta-\alpha), \delta \eta\right\}}} .
$$

Proof Let $x^{*} \in \mathbf{S}\left(\mathcal{C}, F, \mathcal{G}_{A}\right)$. According to Theorem 3.3 and thanks to the condition $\left(\mathcal{H}_{\psi}\right)$, for each $x \in \mathcal{C}$, we obtain

$$
\begin{aligned}
\Delta_{\Omega_{\gamma}^{\varrho}, \delta}^{\psi}(x) & =\inf _{u \in \mathcal{C}}\left\{\Omega_{\gamma}^{\varrho}(u)+\delta \psi(x, u)\right\} \\
& \geq \inf _{u \in \mathcal{C}}\left\{\left(\min _{1 \leq i \leq p}\left\{\sum_{k=1}^{m} a_{i k}\right\} \xi-\gamma(\beta-\alpha)\right)\left\|u-x^{*}\right\|^{2}+\delta \eta\|x-u\|^{2}\right\} \\
& \geq \min \left\{\min _{1 \leq i \leq p}\left\{\sum_{k=1}^{m} a_{i k}\right\} \xi-\gamma(\beta-\alpha), \delta \eta\right\} \inf _{u \in \mathcal{C}}\left\{\left\|u-x^{*}\right\|^{2}+\|x-u\|^{2}\right\} \\
& \geq \frac{1}{2} \min \left\{\min _{1 \leq i \leq p}\left\{\sum_{k=1}^{m} a_{i k}\right\} \xi-\gamma(\beta-\alpha), \delta \eta\right\}\left\|x-x^{*}\right\|^{2} .
\end{aligned}
$$

Hence,

$$
d\left(x, \mathbf{S}\left(\mathcal{C}, F, \mathcal{G}_{A}\right)\right) \leq\left\|x-x^{*}\right\| \leq \sqrt{\frac{2 \Delta_{\Omega_{\gamma}, \delta}^{\psi}(x)}{\min \left\{\min _{1 \leq i \leq p}\left\{\sum_{k=1}^{m} a_{i k}\right\} \xi-\gamma(\beta-\alpha), \delta \eta\right\}}} .
$$

Therefore, the proof is completed. 
Finally, we give an illustrative example where it is shown that all assumptions of Theorem 3.3 and Theorem 3.4 hold.

Example 3.3 Let $X, \mathcal{C}, p, m, A, F, \varrho, \psi, \alpha, \beta, \gamma, \delta, \theta, \eta$ be as in Example 3.2. Then, the solution set of $\operatorname{VEP}\left(\mathcal{C}, F, \mathcal{G}_{A}\right), \mathbf{S}\left(\mathcal{C}, F, \mathcal{G}_{A}\right)=\{0\}$ and the gap functions of the problem $\operatorname{VEP}\left(\mathcal{C}, F, \mathcal{G}_{A}\right)$ are defined by

$$
\Omega_{\gamma}^{\varrho}=3 x^{2}, \Delta_{\Omega_{\gamma}^{\varrho}, \delta}^{\psi}(x)=\frac{3}{7} x^{2} .
$$

Now, we check that all conditions of Theorem 3.3 are fulfilled.

$\left(\mathcal{H}_{F}\right)_{1}:$ We have

$$
\begin{aligned}
\mathcal{K}_{1} & =\left\{x \in \mathcal{C}: \sum_{k=1}^{m} a_{1 k} F_{k}(x, y) \geq 0, \forall y \in \mathcal{C}\right\} \\
& =\left\{x \in\left[0, e^{2}\right]: y^{2}+2 x y-3 x^{2} \geq 0, \forall y \in\left[0, e^{2}\right]\right\} \\
& =\{0\}, \\
\mathcal{K}_{2} & =\left\{x \in \mathcal{C}: \sum_{k=1}^{m} a_{2 k} F_{k}(x, y) \geq 0, \forall y \in \mathcal{C}\right\} \\
& =\left\{x \in\left[0, e^{2}\right]: y^{2}+\frac{5}{2} x y-\frac{7}{2} x^{2} \geq 0, \forall y \in\left[0, e^{2}\right]\right\} \\
& =\{0\} .
\end{aligned}
$$

This implies that $0 \in \bigcap_{i=1}^{2} \mathcal{K}_{i}$, i.e., $\bigcap_{i=1}^{2} \mathcal{K}_{i} \neq \emptyset$.

$\left(\mathcal{H}_{F}\right)_{2}$ : Setting $\xi=2$, for each $(x, y) \in \mathcal{C} \times \mathcal{C}$, we get

$$
\begin{aligned}
F(x, y)+F(y, x)+\xi\|x-y\|^{2} e & =\left(\begin{array}{l}
F_{1}(x, y)+F_{1}(y, x)+2(x-y)^{2} \\
F_{2}(x, y)+F_{2}(y, x)+2(x-y)^{2}
\end{array}\right) \\
& =\left(\begin{array}{c}
-2(x-y)^{2}+2(x-y)^{2} \\
-\frac{5}{2}(x-y)^{2}+2(x-y)^{2}
\end{array}\right) \\
& =\left(\begin{array}{c}
0 \\
-\frac{1}{2}(x-y)^{2}
\end{array}\right) \in-\mathcal{G}_{A} .
\end{aligned}
$$

$\underline{\left(\mathcal{H}_{A}\right)}$ : The matrix $A \in \mathbb{R}^{p \times m}$ with $A=\left(a_{i j}\right)$ satisfies that

$$
\min _{1 \leq i \leq p}\left\{\sum_{k=1}^{m} a_{i k}\right\}=\min \{1,1\}=1>0 .
$$

Furthermore, it follows from Example 3.2 that all assumptions of Theorems 3.1 and 3.2 hold. Thus, we conclude that all the assumptions of Theorems 3.3 and 3.4 are satisfied and so, (18) and (24) hold.

Indeed, for example, for all $x \in\left[0, e^{2}\right]$ and for $x^{*}=0 \in \mathbf{S}\left(\mathcal{C}, F, \mathcal{G}_{A}\right)$, we have

$$
d\left(x, \mathbf{S}\left(\mathcal{C}, F, \mathcal{G}_{A}\right)\right) \leq\left\|x-x^{*}\right\|=x
$$


and

$$
\begin{gathered}
\sqrt{\frac{\Omega_{\gamma}^{\varrho}(x)}{\min _{1 \leq i \leq p}\left\{\sum_{k=1}^{m} a_{i k}\right\} \xi-\gamma(\beta-\alpha)}}=\sqrt{\frac{3 x^{2}}{\frac{3}{2}}}=\sqrt{2} x \geq x, \\
\sqrt{\frac{2 \Delta_{\Omega_{\gamma}, \delta}^{\psi}(x)}{\min \left\{\min _{1 \leq i \leq p}\left\{\sum_{k=1}^{m} a_{i k}\right\} \xi-\gamma(\beta-\alpha), \delta \eta\right\}}}=\sqrt{\frac{\frac{6}{7} x^{2}}{\frac{1}{2}}}=\sqrt{\frac{12}{7}} x \geq x .
\end{gathered}
$$

Hence, inequalities (18) and (24) hold.

Remark 3.4 Since the error bounds in Theorems 3.3 and 3.4 are established by using the regularized gap functions $\Omega_{\gamma}^{\varrho}$ and $\Delta_{\Omega_{\gamma}^{\varrho}, \delta}^{\psi}$, it follows from Remark 3.3 that the results in Theorems 3.3 and 3.4 are new and extend known error bounds in the literature.

\section{Application to a vector network equilibrium problem}

The network equilibrium model was introduced by Wardrop [36] for a transportation network. This model has played a vital role in the traffic network planning and to optimize the traffic control. Based on vector-valued cost functions or multicriteria consideration, many network equilibrium models have been investigated, see e.g., [7,8,28,37] and the references therein. In this section, we consider a formulation of vector network equilibrium problem with partial order provided by a polyhedral cone generated by some matrix, which illustrates the applicability of the abstract results.

Consider a transportation network $\mathbf{G}=(\mathcal{N}, \mathcal{A})$, where $\mathcal{N}$ and $\mathcal{A}$ denote the set of nodes and directed arcs, respectively. Let $\mathcal{W}$ denote the set of origin-destination (O-D) pairs and $\mathcal{P}_{w}, w \in \mathcal{W}$ denotes the set of available paths joining O-D pair $w$. Let $\hat{d}=\left(\hat{d}_{w}\right)_{w \in \mathcal{W}}$ denote the demand vector, where $\hat{d}_{w}$ denotes the demand of traffic flow on O-D pair $w$. For a given path $k \in \mathcal{P}_{w}$, let $f_{k}$ denote the traffic flow on this path and $\mathbf{f}=\left(f_{1}, f_{2}, \ldots, f_{N}\right)^{\top} \in \mathbb{R}^{N}$, where $N=\sum_{w \in \mathcal{W}}\left|\mathcal{P}_{w}\right|$ being $|\cdot|$ the cardinality of $\mathcal{P}_{w}$. The path flow vector $\mathbf{f}$ induces a flow $v_{e}$ on each arc $e \in \mathcal{A}$ given by

$$
v_{e}=\sum_{w \in \mathcal{W}} \sum_{k \in \mathcal{P}_{w}} \delta_{e k} f_{k},
$$

where $\left[\delta_{e k}\right] \in \mathbb{R}^{v \times N}(v=|\mathcal{A}|)$ is the arc path incidence matrix with

$$
\delta_{e k}= \begin{cases}1 & \text { if } \operatorname{arc} e \text { belongs to path } k \\ 0 & \text { otherwise }\end{cases}
$$

Let $\mathbf{v}=\left(v_{1}, v_{2}, \ldots, v_{v}\right)^{\top} \in \mathbb{R}^{v}$ be the vector of arc flow. We say that a path flow $\mathbf{f}$ satisfies demands if $\sum_{k \in \mathcal{P}_{w}} f_{k}=\hat{d}_{w}$ for all $w \in \mathcal{W}$. A flow $\mathbf{f} \geq \mathbf{0}$ satisfying the demand is called a feasible flow. Let

$$
\mathbf{H}=\left\{\mathbf{f} \in \mathbb{R}^{N}: \mathbf{f} \geq \mathbf{0}, \sum_{k \in \mathcal{P}_{w}} f_{k}=\hat{d}_{w}, \forall w \in \mathcal{W}\right\} .
$$

Assume that $\mathbf{H} \neq \varnothing$. It is easy to check that the set $\mathbf{H}$ is compact and convex. Let $\mathbf{c}_{e}: \mathbb{R}^{v} \rightarrow \mathbb{R}^{m}$ be a vector-valued cost function for arc $e$ which is in general a function of all the arc flows. 
We assume that $\mathbf{c}_{e}(\mathbf{v})=\left(\mathbf{c}_{e}^{1}(\mathbf{v}), \mathbf{c}_{e}^{2}(\mathbf{v}), \ldots, \mathbf{c}_{e}^{m}(\mathbf{v})\right)^{\top} \in \mathbb{R}^{m}$. Let $\mathcal{T}_{k}: \mathbb{R}^{N} \rightarrow R^{m}$ be a vectorvalued cost function along the path $k$. For each $w \in \mathcal{W}$ and $k \in \mathcal{P}_{w}$, the vector cost $\mathcal{T}_{k}$ is assumed to be the sum of all the arc cost of the flow $f_{k}$ through arcs, which belong to the path $k$, i.e.,

$$
\mathcal{T}_{k}(\mathbf{f})=\sum_{e \in \mathcal{A}} \delta_{e k} \mathbf{c}_{e}(\mathbf{v})=\left(\begin{array}{c}
\sum_{e \in \mathcal{A}} \delta_{e k} \mathbf{c}_{e}^{1}(\mathbf{v}) \\
\sum_{e \in \mathcal{A}} \delta_{e k} \mathbf{c}_{e}^{2}(\mathbf{v}) \\
\vdots \\
\sum_{e \in \mathcal{A}} \delta_{e k} \mathbf{c}_{e}^{m}(\mathbf{v})
\end{array}\right) .
$$

For each $w \in \mathcal{W}, k \in \mathcal{P}_{w}, j \in\{1,2, \ldots, m\}, \mathbf{v} \in \mathbb{R}^{v}$ and $\mathbf{f} \in \mathbf{H}$, let

$$
\mathcal{T}_{k}^{j}(\mathbf{f})=\sum_{e \in \mathcal{A}} \delta_{e k} \mathbf{c}_{e}^{j}(\mathbf{v}) \text { and } \mathcal{T}^{j}(\mathbf{f})=\left(\mathcal{T}_{1}^{j}(\mathbf{f}), \mathcal{T}_{2}^{j}(\mathbf{f}), \ldots, \mathcal{T}_{N}^{j}(\mathbf{f})\right)^{\top} \in \mathbb{R}^{N}
$$

Then, for each $\mathbf{f} \in \mathbf{H}$, let

$$
\mathcal{T}(\mathbf{f})=\left(\mathcal{T}^{1}(\mathbf{f}), \mathcal{T}^{2}(\mathbf{f}), \ldots, \mathcal{T}^{m}(\mathbf{f})\right)^{\top}=\left(\mathcal{T}_{1}(\mathbf{f}), \mathcal{T}_{2}(\mathbf{f}), \ldots, \mathcal{T}_{N}(\mathbf{f})\right) \in \mathbb{R}^{m \times N} .
$$

Let $\mathcal{G}_{A}$ be the polyhedral cone considered in Sect. 2. We now introduce the notion of $\mathcal{G}_{A}$-equilibria of a flow $\mathbf{f} \in \mathbf{H}$.

Definition 4.1 A flow $\mathbf{f} \in \mathbf{H}$ is said to be in $\mathcal{G}_{A}$-equilibrium if for all $w \in \mathcal{W}, k \in \mathcal{P}_{w}, l \in \mathcal{P}_{w}$,

$$
\mathcal{T}_{k}(\mathbf{f})-\mathcal{T}_{l}(\mathbf{f}) \in \operatorname{int}\left(\mathcal{G}_{A}\right) \Longrightarrow f_{k}=0 .
$$

Remark 4.1 Let $p=m$. If $A$ is the identity matrix of size $m$, then $\mathcal{G}_{A}=\left\{x \in \mathbb{R}^{m}: A x \geq\right.$ 0 $\}=\mathbb{R}_{+}^{m}$. We get that (25) becomes

$$
\mathcal{T}_{k}(\mathbf{f})-\mathcal{T}_{l}(\mathbf{f}) \in \operatorname{int}\left(\mathbb{R}_{+}^{m}\right) \Longrightarrow f_{k}=0 .
$$

Then the flow $\mathbf{f}$ is in weak vector equilibrium, see [7, Definition 3.2].

Proposition 4.1 The path flow $\mathbf{f}^{*} \in \mathbf{H}$ is in $\mathcal{G}_{A}$-equilibrium if $\mathbf{f}^{*}$ solves the vector variational inequality (for short, $\operatorname{VVI}\left(\mathbf{H}, \mathcal{T}, \mathcal{G}_{A}\right)$ ) :

$$
\left\langle\mathcal{T}\left(\mathbf{f}^{*}\right), \mathbf{h}-\mathbf{f}^{*}\right\rangle \notin-\operatorname{int}\left(\mathcal{G}_{A}\right), \quad \forall \mathbf{h} \in \mathbf{H} .
$$

Proof Let $\mathbf{f}^{*} \in \mathbf{H}$ be the solution of problem $\operatorname{VVI}\left(\mathbf{H}, \mathcal{T}, \mathcal{G}_{A}\right)$. Consider a path flow vector $\mathbf{h}$ to be such that

$$
\mathbf{h}_{q}= \begin{cases}\mathbf{f}_{q}^{*} & \text { if } q \neq k \text { or } l \\ 0 & \text { if } q=k \\ \mathbf{f}_{k}^{*}+\mathbf{f}_{l}^{*} & \text { if } q=l .\end{cases}
$$

Hence, $\forall w \in \mathcal{W}, \sum_{q \in \mathcal{P}_{w}} h_{q}=\sum_{q \in \mathcal{P}_{w}} f_{q}^{*}=\hat{d}_{w}$, and so $\mathbf{h} \in \mathbf{H}$. Then, we have

$$
\begin{aligned}
\left\langle\mathcal{T}\left(\mathbf{f}^{*}\right), \mathbf{h}-\mathbf{f}^{*}\right\rangle & =\sum_{w \in \mathcal{W}} \sum_{q \in \mathcal{P}_{w}}\left(h_{q}-f_{q}\right) \mathcal{T}_{q}\left(\mathbf{f}^{*}\right) \\
& =\left(h_{k}-f_{k}^{*}\right) \mathcal{T}_{k}\left(\mathbf{f}^{*}\right)+\left(h_{l}-f_{l}^{*}\right) \mathcal{T}_{l}\left(\mathbf{f}^{*}\right) \\
& =f_{k}^{*}\left(\mathcal{T}_{l}\left(\mathbf{f}^{*}\right)-\mathcal{T}_{k}\left(\mathbf{f}^{*}\right)\right) \notin-\operatorname{int}\left(\mathcal{G}_{A}\right) .
\end{aligned}
$$


Equation (27) is equivalent to

$$
\begin{aligned}
& f_{k}^{*}\left(\sum_{j=1}^{m} a_{1 j}\left(\mathcal{T}_{l}^{j}\left(\mathbf{f}^{*}\right)-\mathcal{T}_{k}^{j}\left(\mathbf{f}^{*}\right)\right), \sum_{j=1}^{m} a_{2 j}\left(\mathcal{T}_{l}^{j}\left(\mathbf{f}^{*}\right)-\mathcal{T}_{k}^{j}\left(\mathbf{f}^{*}\right)\right), \ldots, \sum_{j=1}^{m} a_{p j}\left(\mathcal{T}_{l}^{j}\left(\mathbf{f}^{*}\right)-\mathcal{T}_{k}^{j}\left(\mathbf{f}^{*}\right)\right)\right)^{\top} \\
& \quad \times \notin-\operatorname{int}\left(\mathbb{R}_{+}^{p}\right) .
\end{aligned}
$$

If $\mathcal{T}_{k}\left(\mathbf{f}^{*}\right)-\mathcal{T}_{l}\left(\mathbf{f}^{*}\right) \in \operatorname{int}\left(\mathcal{G}_{A}\right)$, i.e.,

$$
\begin{aligned}
& \left(\sum_{j=1}^{m} a_{1 j}\left(\mathcal{T}_{l}^{j}\left(\mathbf{f}^{*}\right)-\mathcal{T}_{k}^{j}\left(\mathbf{f}^{*}\right)\right), \sum_{j=1}^{m} a_{2 j}\left(\mathcal{T}_{l}^{j}\left(\mathbf{f}^{*}\right)-\mathcal{T}_{k}^{j}\left(\mathbf{f}^{*}\right)\right), \ldots, \sum_{j=1}^{m} a_{p j}\left(\mathcal{T}_{l}^{j}\left(\mathbf{f}^{*}\right)-\mathcal{T}_{k}^{j}\left(\mathbf{f}^{*}\right)\right)\right)^{\top} \\
& \quad \times \in-\operatorname{int}\left(\mathbb{R}_{+}^{p}\right),
\end{aligned}
$$

then, it follows from (28) and (29) that $f_{k}^{*}=0$. Thus, the path flow $\mathbf{f}^{*} \in \mathbf{H}$ is in $\mathcal{G}_{A^{-}}$ equilibrium.

We always assume that the solution set of the problem $\operatorname{VVI}\left(\mathbf{H}, \mathcal{T}, \mathcal{G}_{A}\right), \mathbf{S}\left(\mathbf{H}, \mathcal{T}, \mathcal{G}_{A}\right)$, is nonempty. Now, we impose the following hypotheses on the the data of the problem $\operatorname{VVI}\left(\mathbf{H}, \mathcal{T}, \mathcal{G}_{A}\right)$ :

$\left(\mathcal{H}_{\mathcal{T}}\right)_{1}: \bigcap_{i=1}^{p} \widetilde{\mathcal{K}}_{i}:=\left\{\mathbf{f} \in \mathbf{H}: \sum_{j=1}^{m} a_{i j} \sum_{w \in \mathcal{W}} \sum_{k \in \mathcal{P}_{w}}\left(h_{k}-f_{k}\right) \mathcal{T}_{k}^{j}(\mathbf{f}) \geq 0, \forall \mathbf{h} \in \mathbf{H}\right\} \neq \emptyset ;$

$\left(\mathcal{H}_{\mathcal{T}}\right)_{2}$ : There exists $\widetilde{\xi}>0$ if, for each $(\mathbf{f}, \mathbf{h}) \in \mathbf{H} \times \mathbf{H}$,

$$
\sum_{w \in \mathcal{W}} \sum_{k \in \mathcal{P}_{w}}\left[\left(h_{k}-f_{k}\right) \mathcal{T}_{k}(\mathbf{f})+\left(f_{k}-h_{k}\right) \mathcal{T}_{k}(\mathbf{h})\right]+\tilde{\xi}\|\mathbf{f}-\mathbf{h}\|^{2} e \in-\mathcal{G}_{A}
$$

where $e=(1,1, \ldots, 1)^{\top} \in \mathbb{R}^{m}$;

$\left(\mathcal{H}_{\mathcal{T}}\right)_{3}$ : the function $\mathcal{T}_{k}^{j}(\cdot)$ is continuous for all $w \in \mathcal{W}, k \in \mathcal{P}_{w}$ and $j \in\{1, \ldots, m\}$.

For each fixed constant $\gamma>0$, we consider the following function $\widetilde{\Omega}_{\gamma}^{\varrho}: \mathbf{H} \rightarrow \mathbb{R}$ defined by

$$
\begin{aligned}
\widetilde{\Omega}_{\gamma}^{\varrho}(\mathbf{f}) & =\sup _{\mathbf{h} \in \mathbf{H}}\left(-\max _{1 \leq i \leq p}\left\{\sum_{j=1}^{m} a_{i j}\left\langle\mathcal{T}^{j}(\mathbf{f}), \mathbf{h}-\mathbf{f}\right\rangle\right\}-\gamma \varrho(\mathbf{f}, \mathbf{h})\right) \\
& =\sup _{\mathbf{h} \in \mathbf{H}}\left(-\max _{1 \leq i \leq p}\left\{\sum_{j=1}^{m} a_{i j} \sum_{w \in \mathcal{W}} \sum_{k \in \mathcal{P}_{w}}\left(h_{k}-f_{k}\right) \mathcal{T}_{k}^{j}(\mathbf{f})\right\}-\gamma \varrho(\mathbf{f}, \mathbf{h})\right),
\end{aligned}
$$

where $\varrho: \mathbf{H} \times \mathbf{H} \rightarrow \mathbb{R}_{+}$is a continuously differentiable function, which satisfies the condition $\left(\mathcal{H}_{\varrho}\right)$.

Theorem 4.1 Under the hypotheses $\left(\mathcal{H}_{\mathcal{T}}\right)_{1},\left(\mathcal{H}_{\mathcal{T}}\right)_{2},\left(\mathcal{H}_{A}\right)$ and $\left(\mathcal{H}_{\varrho}\right)$, we have

(i) for each $\gamma>0$, the function $\widetilde{\Omega}_{\gamma}^{\varrho}: \mathbf{H} \rightarrow \mathbb{R}$ defined by (30) is a regularized gap function for $\operatorname{VVI}\left(\mathbf{H}, \mathcal{T}, \mathcal{G}_{A}\right)$.

(ii) if $\gamma>0$ is such that

$$
\min _{1 \leq i \leq p}\left\{\sum_{k=1}^{m} a_{i k}\right\} \widetilde{\xi}-\gamma(\beta-\alpha)>0
$$

then, for each $\mathbf{f} \in \mathbf{H}$, it holds

$$
d\left(\mathbf{f}, \mathbf{S}\left(\mathbf{H}, \mathcal{T}, \mathcal{G}_{A}\right)\right) \leq \sqrt{\frac{\widetilde{\Omega}_{\gamma}^{\varrho}(\mathbf{f})}{\min _{1 \leq i \leq p}\left\{\sum_{j=1}^{m} a_{i j}\right\} \widetilde{\xi}-\gamma(\beta-\alpha)}} .
$$


Proof For each $j \in\{1,2, \ldots, m\}$, we consider the functions $F_{j}: \mathbf{H} \times \mathbf{H} \rightarrow \mathbb{R}$ and $F: \mathbf{H} \times$ $\mathbf{H} \rightarrow \mathbb{R}^{m}$ defined by

$$
\begin{aligned}
F_{j}(\mathbf{f}, \mathbf{h}) & =\left\langle\mathcal{T}^{j}(\mathbf{f}), \mathbf{h}-\mathbf{f}\right\rangle=\sum_{w \in \mathcal{W}} \sum_{k \in \mathcal{P}_{w}}\left(h_{k}-f_{k}\right) \mathcal{T}_{k}^{j}(\mathbf{f}), \\
F(\mathbf{f}, \mathbf{h}) & =\left(F_{1}(\mathbf{f}, \mathbf{h}), F_{2}(\mathbf{f}, \mathbf{h}), \ldots, F_{m}(\mathbf{f}, \mathbf{h})\right)^{\top} \in \mathbb{R}^{m} \quad \forall \mathbf{f}, \mathbf{h} \in \mathbf{H} .
\end{aligned}
$$

Then, the problem $\operatorname{VVI}\left(\mathbf{H}, \mathcal{T}, \mathcal{G}_{A}\right)$ is equivalent to the problem $\operatorname{VEP}\left(\mathbf{H}, F, \mathcal{G}_{A}\right)$, i.e., the function $\widetilde{\Omega}_{\gamma}^{\varrho}$ is equivalent to the function $\Omega_{\gamma}^{\varrho}$. Since $\mathbf{H} \ni \mathbf{h} \mapsto F_{j}(\mathbf{f}, \mathbf{h})$ is an affine function for all $j \in\{1,2, \ldots, m\}$, it is easily seen that $F$ is $\mathcal{G}_{A}$-convex in the second component. Moreover, an easy computation shows that conditions $\left(\mathcal{H}_{F}\right)_{1}$ and $\left(\mathcal{H}_{F}\right)_{2}$ are satisfied with $\mathcal{C}=\mathbf{H}, \mathcal{K}_{i}=\widetilde{\mathcal{K}}_{i}$ for all $i \in\{1,2, \ldots, p\}$ and $\xi=\widetilde{\xi}$. Therefore, by applying Theorems 3.1 and 3.3, we conclude that Theorem 4.1 holds.

Remark 4.2 It follows from the condition $\left(\mathcal{H}_{\mathcal{T}}\right)_{3}$ that $F_{j}$ is continuous on $\mathbf{H} \times \mathbf{H}$ for all $j \in\{1,2, \ldots, m\}$. Thus, if conditions $\left(\mathcal{H}_{\mathcal{T}}\right)_{3}$ and $\left(\mathcal{H}_{\varrho}\right)$ hold, then by Lemma 3.1 the function $\widetilde{\Omega}_{\gamma}^{\varrho}$ is continuous.

Next, we consider a gap function based on the Moreau-Yosida regularization of $\widetilde{\Omega}_{\gamma}^{\varrho}: \mathbf{H} \rightarrow$ $\mathbb{R}$ as follows:

$$
\widetilde{\Delta}_{\widetilde{\Omega}_{\gamma}^{\varrho}, \delta}^{\psi}(\mathbf{f})=\inf _{\mathbf{g} \in \mathbf{H}}\left\{\widetilde{\Omega}_{\gamma}^{\varrho}(\mathbf{g})+\delta \psi(\mathbf{f}, \mathbf{g})\right\}
$$

where $\psi: \mathbf{H} \times \mathbf{H} \rightarrow \mathbb{R}_{+}$is a continuously differentiable function, which satisfies the condition $\left(\mathcal{H}_{\psi}\right)$. From (30) and (31), we can rewrite the function $\widetilde{\Delta}_{\widetilde{\Omega}_{\gamma}{ }^{\rho}, \delta}$ as follows:

$$
\begin{aligned}
& \widetilde{\Delta}_{\widetilde{\Omega}_{\gamma}^{\varrho}, \delta}^{\psi}(\mathbf{f}) \\
& =\inf _{\mathbf{g} \in \mathbf{H}}\left\{\sup _{\mathbf{h} \in \mathbf{H}}\left(-\max _{1 \leq i \leq p}\left\{\sum_{j=1}^{m} a_{i j} \sum_{w \in \mathcal{W}} \sum_{k \in \mathcal{P}_{w}}\left(h_{k}-g_{k}\right) \mathcal{T}_{k}^{j}(\mathbf{g})\right\}-\gamma \varrho(\mathbf{g}, \mathbf{h})\right)+\delta \psi(\mathbf{f}, \mathbf{g})\right\}
\end{aligned}
$$

From Theorems 3.2, 3.4 and 4.1, we directly obtain the following error estimates based on the Moreau-Yosida regularized gap function for $\operatorname{VVI}\left(\mathbf{H}, \mathcal{T}, \mathcal{G}_{A}\right)$.

Theorem 4.2 Under the hypotheses $\left(\mathcal{H}_{\mathcal{T}}\right)_{1}-\left(\mathcal{H}_{\mathcal{T}}\right)_{3},\left(\mathcal{H}_{A}\right),\left(\mathcal{H}_{\varrho}\right)$ and $\left(\mathcal{H}_{\psi}\right)$, we have

(i) for any $\eta, \zeta>0$, the function $\widetilde{\Omega}_{\gamma}^{\varrho}: \mathbf{H} \rightarrow \mathbb{R}$ defined by (32) is the Moreau-Yosida regularized gap function for $\operatorname{VVI}\left(\mathbf{H}, \mathcal{T}, \mathcal{G}_{A}\right)$.

(ii) for any $\eta>0$, if $\gamma>0$ is such that

$$
\min _{1 \leq i \leq p}\left\{\sum_{k=1}^{m} a_{i k}\right\} \widetilde{\xi}-\gamma(\beta-\alpha)>0,
$$

then, for each $\mathbf{f} \in \mathbf{H}$, it holds

$$
d\left(\mathbf{f}, \mathbf{S}\left(\mathbf{H}, \mathcal{T}, \mathcal{G}_{A}\right)\right) \leq \sqrt{\frac{2 \widetilde{\Delta}_{\widetilde{\Omega}_{\gamma}, \delta}^{\psi}(\mathbf{f})}{\min \left\{\min _{1 \leq i \leq p}\left\{\sum_{k=1}^{m} a_{i k}\right\} \widetilde{\xi}-\gamma(\beta-\alpha), \delta \eta\right\}}}
$$


Remark 4.3 (i) To the best of our knowledge, up to now, there is no paper concerning regularized gap functions and error bounds for vector network equilibrium problem with partial order provided by a polyhedral cone generated by some matrix. Thus, Theorems 4.1 and 4.2 are new.

(ii) In the case of Remark 4.1, $\mathcal{G}_{A}=\left\{x \in \mathbb{R}^{m}: A x \geq \mathbf{0}\right\}=\mathbb{R}_{+}^{m}$. Then, our formulated vector network equilibrium problem reduces to the vector network equilibrium problem with the ordering cone $\mathbb{R}_{+}^{m}$ considered in $[7,8]$. In this special case, our main results in this section are still new.

\section{Conclusions}

In this work, we have studied a class of vector equilibrium problems with partial order provided by a polyhedral cone generated by some matrix $A$. Using the concept of $\mathcal{G}_{A}$-convexity of a vector-valued function, we have proposed some gap functions in forms of the Fukushima regularization and the Moreau-Yosida regularization to the problem $\operatorname{VEP}\left(\mathcal{C}, F, \mathcal{G}_{A}\right)$ (Theorems 3.1 and 3.2). We have also provided some error bounds for the problem $\operatorname{VEP}\left(\mathcal{C}, F, \mathcal{G}_{A}\right)$ by virtue of these regularized gap functions (Theorems 3.3 and 3.4). Especially, to illustrate our main theoretical findings in a real-world application, we have derived a vector network equilibrium problem in Sect. 4. Our results in this section are new even in the case where the ordering cone in $\mathbb{R}_{+}^{m}$.

Acknowledgements This work for the second author was partially supported by the Ministerio de Ciencia, Innovación y Universidades (MCIU), Agencia Estatal de Investigación (AEI) (Spain) and Fondo Europeo de Desarrollo Regional (FEDER) under project PGC2018-096899-B-I00 (MCIU/AEI/FEDER, UE). The third author is also supported by the Ministry of Education and Training of Vietnam under Grant No. B2021.SPD.03. The authors are heartily grateful to the anonymous referees for their valuable remarks which greatly improved the results and presentation of the paper.

Funding Open Access funding provided thanks to the CRUE-CSIC agreement with Springer Nature.

Open Access This article is licensed under a Creative Commons Attribution 4.0 International License, which permits use, sharing, adaptation, distribution and reproduction in any medium or format, as long as you give appropriate credit to the original author(s) and the source, provide a link to the Creative Commons licence, and indicate if changes were made. The images or other third party material in this article are included in the article's Creative Commons licence, unless indicated otherwise in a credit line to the material. If material is not included in the article's Creative Commons licence and your intended use is not permitted by statutory regulation or exceeds the permitted use, you will need to obtain permission directly from the copyright holder. To view a copy of this licence, visit http://creativecommons.org/licenses/by/4.0/.

\section{References}

1. Anh, L.Q., Hung, N.V., Tam, V.M.: Regularized gap functions and error bounds for generalized mixed strong vector quasiequilibrium problems. Comput. Appl. Math. 37, 5935-5950 (2018)

2. Auslender, A.: Optimisation: Méthodes Numériques. Masson, Paris (1976). ((in French))

3. Bigi, G., Passacantando, M.: Gap functions for quasi-equilibria. J. Global Optim. 66, 791-810 (2016)

4. Blum, E., Oettli, W.: From optimization and variational inequalities to equilibrium problems. Math. Stud. 63, 123-145 (1994)

5. Burns, F., Fiedler, M., Haynsworth, E.: Polyhedral cones and positive operators. Linear Algebra Appl. 8, 547-559 (1974)

6. Chegancas, J., Burgat, C.: Polyhedral cones associated to $M$-matrices and stability of time varying discrete time systems. J. Math. Anal. Appl. 118, 88-96 (1986) 
7. Chen, G.Y., Goh, C.J., Yang, X.Q.: Vector network equilibrium problems and nonlinear scalarization methods. Math. Methods Oper. Res. 49, 239-253 (1999)

8. Chen, G.Y., Huang, X., Yang, X.: Vector Optimization: Set-valued and Variational Analysis. Lecture Notes in Econom. and Math. Systems 541. Springer-Verlag, Berlin, (2005)

9. Daniele, P., Giannessi, F., Maugeri, A. (Eds.): Equilibrium problems and variational models. Nonconvex Optim. Appl. 68. Kluwer Academic Publishers, Norwell (2003)

10. Eichfelder, G.: Adaptive Scalarization Methods in Multiobjective Optimization. Springer, Berlin (2008)

11. Fan, K.: A minimax inequality and its application. In: O. Shisha (Ed.): Inequalities III (Proc. Third Sympos., Univ. California, Los Angeles, Calif., 1969), 103-113. Academic, New York (1972)

12. Fukushima, M.: Equivalent differentiable optimization problems and descent methods for asymmetric variational inequality problems. Math. Program. 53, 99-110 (1992)

13. Giannessi, F., Maugeri, F.A., Pardalos P.M. (Eds.): Equilibrium problems: nonsmooth optimization and variational inequality models (Vol. 58), Springer Science \& Business Media (2006)

14. Goldman, A.J., Tucker, A.W.: Polyhedral Convex Cones in Linear inequalities and Related Systems. In: Kuhn, H.W., Tucker, A.W. (Eds.): Princeton U. P., Princeton, New Jersey (1956)

15. Gutiérrez, C., Huerga, L., Jiménez, B., Novo, V.: Optimality conditions for approximate proper solutions in multiobjective optimization with polyhedral cones. TOP 28, 526-544 (2020)

16. Gutiérrez, C., Huerga, L., Novo, V.: Nonlinear scalarization in multiobjective optimization with a polyhedral ordering cone. Int. Trans. Oper. Res. 25, 763-779 (2018)

17. Hai, L.P., Huerga, L., Khanh, P.Q., Novo, V.: Variants of the Ekeland variational principle for approximate proper solutions of vector equilibrium problems. J. Global Optim. 74, 361-382 (2019)

18. Hung, N.V.: On the stability of the solution mapping for parametric traffic network problems. Indag. Math. 29, 885-894 (2018)

19. Hung, N.V., Tam, V.M., Liu, Z., Yao, J.C.: A novel approach to Hölder continuity of a class of parametric variational-hemivariational inequalities. Oper. Res. Lett. 49, 283-289 (2021)

20. Hung, N.V., Tam, V.M., Tuan, N.H., O’Regan, D.: Convergence analysis of solution sets for fuzzy optimization problems. J. Comput. Appl. Math. 369, 112615 (2020)

21. Hung, N.V., Migórski, S., Tam, V.M., Zeng, S.D.: Gap functions and error bounds for variationalhemivariational inequalities. Acta. Appl. Math. 169, 691-709 (2020)

22. Hung, N.V., Tam, V.M., Pitea, A.: Global error bounds for mixed quasi-hemivariational inequality problems on Hadamard manifolds. Optimization 69, 2033-2052 (2020)

23. Hung, N.V., Tam, V.M., Zhou, Y.: A new class of strong mixed vector GQVIP-generalized quasi-variational inequality problems in fuzzy environment with regularized gap functions based error bounds. J. Comput. Appl. Math. 381, 113055 (2021)

24. Hung, N.V.: Generalized Levitin-Polyak well-posedness for controlled systems of FMQHI-fuzzy mixed quasi-hemivariational inequalities of Minty type. J. Comput. Appl. Math. 386, 113263 (2021)

25. Hung, N.V., Keller, A.A.: Painlevé-Kuratowski convergence of the solution sets for controlled systems of fuzzy vector quasi-optimization problems with application to controlling traffic networks under uncertainty. Comput. Appl. Math. 40, 1-21 (2021)

26. Kassay, G., Rădulescu, V.: Equilibrium Problems and Applications. Academic Press, Cambridge (2018)

27. Khan, S.A., Chen, J.W.: Gap functions and error bounds for generalized mixed vector equilibrium problems. J. Optim. Theory Appl. 166, 767-776 (2015)

28. Magnanti, T.L.: Models and algorithms for predicting urban traffic equilibria. In: Florian, M. (ed.) Transportation Planning Models. Elsevier, Amsterdam (1984)

29. Mastroeni, G.: Gap functions for equilibrium problems. J. Global Optim. 27, 411-426 (2003)

30. Muu, L.D., Oettli, W.: Convergence of an adaptive penalty scheme for finding constrained equilibria. Nonlinear Anal. TMA 18, 1159-1166 (1992)

31. Rahmo, E.D., Stasiak, A., Studniarski, M.: Lower and upper Ginchev derivatives of vector functions and their applications to multiobjective optimization. Optim. Lett. 8, 653-667 (2014)

32. Rockafellar, R.T., Wets, R.J.-B.: Variational Analysis. Springer, Berlin (2009)

33. Stasiak, A.: Higher-order conditions for strict local Pareto minima for problems with partial order introduced by a polyhedral cone. J. Appl. Anal. 24, 45-54 (2018)

34. Stoer, J., Witzgall, C.: Convexity and Optimization in Finite Dimensions I. Springer, Berlin (1970)

35. Sun, X.K., Chai, Y.: Gap functions and error bounds for generalized vector variational inequalities. Optim. Lett. 8, 1663-1673 (2014)

36. Wardrop, J.: Some theoretical aspects of road traffic research. Proceed. Inst. Civil Eng. Part II 1, 325-378 (1952)

37. Wei, H., Chen, C., Wu, B.: Vector network equilibrium problems with uncertain demands and capacity constraints of arcs. Optim. Lett. 15, 1113-1131 (2021)

38. Xia, X.: Zero polyhedral cones. Appl. Math. Lett. 16, 961-966 (2003) 
39. Yamashita, N., Fukushima, M.: Equivalent unconstraint minimization and global error bounds for variational inequality problems. SIAM J. Control Optim. 35, 273-284 (1997)

40. Zhang, X., Ma, F., Wang, Y.: A Newton-type algorithm for generalized linear complementarity problem over a polyhedral cone. Appl. Math. Comput. 169, 388-401 (2005)

Publisher's Note Springer Nature remains neutral with regard to jurisdictional claims in published maps and institutional affiliations. 